Journal of Accident and Emergency Medicine 1994 11, 17-19

\title{
Protective clothing for accident and emergency personnel
}

\author{
D.J. STEEDMAN
}

Department of Accident and Emergency Medicine, The Royal Infirmary, Lauriston Place, Edinburgh

\section{SUMMARY}

There is a significant risk of clothing soilure and skin contamination from patients' blood or other body fluids whilst working in an accident and emergency (A\&E) department. It is therefore unhygienic to wear personal clothing and traditional uniforms do not provide adequate protection. Contamination occurs despite operating 'universal precautions' and emergency presentations often preclude adopting such precautions despite the anticipation of possible contact with blood or other body fluids.

The protection afforded to medical staff working in an A\&E department by a suit made from a liquid repellent polyester fabric was assessed during the period 2 November 1992-1 January 1993. Ninetyone splash incidents were recorded. A total of $85.7 \%$ of splashes (78) were with patients' blood, $13.1 \%$ with vomitus (12) and $1.1 \%$ with pus (1). There were no instances of splashes to the suit that resulted in strike through to the inner surface or visible contamination of underlying skin. However, some $15.4 \%$ of splashes (14) resulted in contamination of exposed skin ad $78.6 \%$ of these (11) occurred between glove and sleeve.

Clothing of appropriated design and fabric can afford skin protection from blood and body fluid contamination. Such clothing alone does not provide overall protection and other precautions currently recommended should be taken.

Key words: clothing, contamination, protection transmissable infective agents.

\section{INTRODUCTION}

Correspondence: D.J. Steedman, Department of Accident and Emergency Medicine, The Royal Infirmary, Lauriston Place, Edinburgh EH3 9YW
Increasing concern about the risks of occupational exposure to HIV and hepatitis viruses has led to recommendations on precautions against exposure to patient's blood or body fluids. ${ }^{1}$ These practical guidelines include taking simple protective measures whilst working in all environments to avoid contamination of skin and clothing with blood.
Most A\&E departments operate a policy of 'universal precautions' with medical and nursing staff receiving guidance in appropriate barrier procedures when contact with blood or other body fluids is anticipated. ${ }^{2}$ Despite the appropriate use of gloves, masks, face shields, gowns and plastic aprons, splashes on clothing and skin contamination may occur.

Doctors working in UK A\&E departments generally still wear white coats over personal clothing and nurses continue to wear traditional ward uniforms. In a previous study of 212 splash incidents from patients' blood and other body fluids, $47.6 \%$ of splashes (101) resulted in skin contamination and $28.7 \%$ of these (29) occurred despite the area being covered by personal clothing or uniform. ${ }^{3}$ The soilure of personal clothing is both unacceptable and unhygienic. Contamination of skin despite wearing a uniform suggests that the design and the material used are inappropriate. Nursing uniforms do not protect the legs or arms, and white coats do not protect against spattering of personal clothing. White coats have also been shown to be a potential source of cross infection, especially in A\&E departments. ${ }^{4}$

An NHS report on the choice of reusable fabrics for operating theatres places emphasis on certain performance specifications which should meet British Standards. A new generation of ultrafine polyester fabrics are now available which meet these criteria. These fabrics are liquid repellent and therefore should act as a barrier against liquid borne trans. missible infective agents.

This study reports on a suit made from this type of fabric designed to afford protection in an emergency care environment.

\section{METHODS}

The suit comprised a long-sleeved tunic and trousers with occlusive cuffs at the wrists and ankles. Design features of the suit focused on providing protection 
to the arms, front of the trunk and legs reflecting the pattern of skin contamination identified in a previous study. ${ }^{3}$ Laundered and packaged tunics and trousers were provided for each A\&E doctor at the start of their shift. Doctors were asked to examined both their suit and any exposed skin after each patient contact and if there was definite soilure of the suit to check for visible evidence of underlying skin contamination. A pro forma was completed for each splash incident.

\section{RESULTS}

During the period 2 November 1992-1 January 1993, 14099 patient attendances were recorded and a total of 91 splash incidents occurred. Although $2.5 \%$ of patients (355) were treated within the resuscitation room, $47.2 \%$ of splash incidents (43) occurred there. A total of $26.4 \%$ of splashes (24) occurred in the examination room and $26.4 \%$ in the trolley room (24). A total of $85.7 \%$ of splashes $(78)$ were with patients' blood, $13.1 \%$ with vomitus (12) and $1.1 \%$ with pus (1). The sites of splashes included: the sleeves in $45 \%$ of incidents (41); the front of the tunic in $47.2 \%$ of incidents; (43) and the trousers in $26.4 \%$ of incidents (24). In $81.3 \%$ of incidents (74), additional protection was being worn (e.g. gloves, masks and eye protection). Personal footwear was soiled in $22 \%$ of incidents (20).

There were no recorded instances of splashes to the suit which resulted in strike through to the inner surface or visible contamination of underlying skin. However, some $15.4 \%$ of splashes (14) resulted in contamination of exposed skin and $78.6 \%$ of these (11) occurred between glove and sleeve.

\section{DISCUSSION}

New European community health and safety directives have resulted in regulations which came into force on January 1993, which require all employers to provide suitable protective equipment to all employees when risks cannot be controlled adequately by other means. ${ }^{6}$ When such equipment is deemed necessary the employer must ensure that it is appropriate for the risks intended.

There is a definite risk of infection following exposure of staff to patients' blood and body fluids. Following unlinked anonymous testing of blood samples from 519 patients admitted to the resuscitation room of this department from October 1990-March 1991, 0.9\% were seropositive for either HIV antibody or Hepatitis $B$ surface antigen. ${ }^{7}$

In most A\&E departments the core of resusciation teams is drawn from the staff working within the unit, and by the very nature of emergency presentation there is often little time to protect personal clothing and exposed skin. Not only are invasive procedures carried out in the resuscitation room but wound management and venous and arterial puncture carried out elsewhere in the department results in appreciable risk of contamination. Nevertheless, it is impossible to anticipate all potential incidents, and staff are often unaware of contamination when it does occur.

This study demonstrates that clothing of appropriate design and fabric can afford skin protection from blood and body fluid contamination. However, it is clear that such clothing alone does not provide overall protection. Therefore, other precautions currently recommended for health care workers to avoid exposure to blood or body fluids should be taken. ${ }^{1}$ Soilure of personal footwear is also both unacceptable and unhygienic and therefore appropriate provision should be made. Following this study, the design of the suit has been modified to minimize the risk of contamination to exposed skin caused by the sleeve rolling up from the glove. Failure of doctors to roll down their sleeves prior to clinical intervention contributed to this exposure and correct procedure has since been re-emphasized.

The clothing utilized a lightweight woven fabric which was 'breatheable'. It was considered comfortable, overcoming the problems encountered with non-woven paper disposable products or clothing made from fabric with a waterproof membrane. The liquid repellence of the fabric is a property of the dense weave and is preserved despite repeated washing.

Although only members of A\&E medical staff participated in this study there is a strong case for recommending that all disciplines engaged in emergency care should wear similar protective clothing. Such staff include A\&E nurses, all doctors with duties in the A\&E department, members of cardiac arrest and trauma teams, and the staff of intensive care, endoscopy and maternity units. The complacency of surgeons' acceptance of arm contamination through pervious gowns during laparotomy has also been criticized. $^{8}$ 
As a result of the successful demonstration of the protective qualities of these suits in an A\&E clinical setting, they have now been adopted as the standard clothing for both medical and nursing staff working in this department.

Employers should seriously consider the introduction of such clothing systems when reviewing hazards and risks of viral transmission from patient to staff working in emergency care environments. Failure to do so may lead to breach of European Community legislation. ${ }^{9}$

\section{ACKNOWLEDGEMENTS}

The author would like to thank: Lojigma International Limited, UK; Rotecno AG, Switzerland for the Rotecno ${ }^{\circledR}$ medical fabric; and Initial UK Limited for workwear management services during this study.

\section{REFERENCES}

1. Joint Working Party of the Hospital Infection Society and the Surgical Infection Study Group (1992) Risk to surgeons and patients from HIV and hepatitis: guidelines on precautions and management of exposure to blood or body fluids. British Medical Journal 305, 1337-1343.

2. Centers for Disease Control (1987) Recommendations for prevention of HIV transmission in health care settings. Morbidity and Mortality Weekly Report, 36, suppl 2S, 5-6S.

3. Littlechild P., Macmillan A., White M.M. \& Steedman D.J. (1992) Contamination of skin and clothing of accident and emergency personnel. British Medical Journal 305, 156-157.

4. Wong D., Nye K. \& Hollis P. (1991) Microbial flora on doctors' white coats. British Medical Journal 303, 1602-1604.

5. NHS Procurement Directorate (1989) Choice of Reusable Fabrics for Operating Theatres (NHS reports STD/89/50). Department of Health, London.

6. Health and Safety Executive (1992a) The Personal Protective Equipment at Work Regulations 1992, No 2966. HMSO, London.

7. Cusack S., Luke L.C., Evans R., Gordon M., Robertson C. \& Peutherer J. (1991) HIV antibody and Hepatitis B surface antigen positivity in a group of accident and emergency resuscitation room patients (Abstract). Archives of Emergency Medicine 9, 82.

8. Wastell C. (1992) Glove punctures and wet gowns: no room for complacency. Annals of the Royal College of Surgeons of England 74, 305.

9. Health and Safety Executive (1992b) The Management of Health and Safety at Work Regulations 1992, No 2051. HMSO, London. 